\title{
Exposure to Flame Retardant Chemicals and Occurrence and Severity of Papillary Thyroid Cancer: A Case-Control Study
}

Kate Hoffman, ${ }^{1}$ Amelia Lorenzo, ${ }^{1}$ Craig M. Butt, ${ }^{1}$ Stephanie C. Hammel,,${ }^{1}$ Brittany Bohinc Henderson, ${ }^{2}$ Sanziana A. Roman, ${ }^{3}$ Randall P. Scheri, ${ }^{3}$ Heather M. Stapleton, ${ }^{1}$ Julie Ann Sosa ${ }^{4}$

${ }^{1}$ Nicholas School of the Environment, Duke University, Durham NC 27708; ${ }^{2}$ Division of Endocrinology, Diabetes, and Metabolism, Department of Internal Medicine, Wake Forest University Baptist Medical Center and Wake Forest Comprehensive Cancer Center, WinstonSalem, NC 27157; ${ }^{3}$ School of Medicine and Department of Surgery, Duke University Medical Center, Durham, NC 27710; ${ }^{4}$ Departments of Surgery and Medicine, Duke Cancer Institute and Duke Clinical Research Institute, Duke University Medical Center, Durham, NC 27710

Short Title: Flame Retardant Chemicals and Thyroid Cancer

\section{Correspondence:}

Julie Ann Sosa

Box DUMC 2945,

Durham, NC 27710

Tel +1 919-668-1767

Fax +1 919-684-6044

Email julie.sosa@duke.edu

\section{Highlights:}

- Exposure to flame retardants was measured for PTC patients and matched controls.

- PTC patients had higher levels of some flame retardants in their homes.

- Associations with FRs varied based on tumor aggressiveness and mutation status.

Key Words: Flame Retardant Chemicals; BDE-209; tris(2-chloroethyl) phosphate (TCEP); Papillary Thyroid Cancer; BRAF V600E

Acknowledgements: We thank Kristen Lynam and Marlo Evans for their tremendous efforts in recruiting participants and coordinating study-related activities. We also gratefully acknowledge the study participants. Funding for this work was provided by Fred \& Alice Stanback, the Duke Cancer Institute, and the Nicholas School of the Environment.

Competing interests declaration: JAS, Member of the Data Monitoring Committee Medullary Thyroid Cancer Consortium Registry supported by NovoNordisk, GlaxoSmithKline, Astra Zeneca, and Eli Lilly; KH, AML, CMB, SCH, BBH, RPS, SAR and HMS have nothing to disclose. 


\section{Abstract:}

Background: Thyroid cancer is the fastest increasing cancer in the U.S., and papillary thyroid cancer (PTC) accounts for $>90 \%$ of incident cases. Increasing exposure to flame retardant chemicals (FRs) has raised concerns about their possible role in this 'epidemic'. The current study was designed to test the hypothesis that higher exposure to FRs is associated with increased odds of PTC.

Methods: PTC patients at the Duke Cancer Institute were approached and invited to participate. Age- and gender-matched controls were recruited from the Duke Health System and surrounding communities. Because suitable biomarkers of long-term exposure do not exist for many common FRs, and levels of FRs in dust are significantly correlated with exposure, relationships between FRs in household dust and PTC were evaluated in addition to available biomarkers. PTC status, measures of aggressiveness (e.g. tumor size) and BRAF V600E mutation were included as outcomes.

Results: Higher levels of some FRs, particularly decabromodiphenyl ether (BDE-209) and tris(2chloroethyl) phosphate in dust, were associated with increased odds of PTC. Participants with dust BDE-209 concentrations above the median level were 2.29 times as likely to have PTC [95\% confidence interval: 1.03, 5.08] compared to those with low BDE-209 concentrations. Associations varied based on tumor aggressiveness and mutation status; TCEP was more strongly associated with larger, more aggressive tumors and BDE-209 was associated with smaller, less aggressive tumors.

Conclusions: Taken together, these results suggest exposure to FRs in the home, particularly BDE-209 and TCEP, may be associated with PTC occurrence and severity, and warrant further study. 
The incidence of thyroid cancer has dramatically increased world-wide over the last several decades (Ho et al. 2015). In the United States, thyroid cancer incidence has increased by an average of $3 \%$ per year over the last four decades, making thyroid cancer one of the fastest increasing cancer among both American women and men (Chen et al. 2009; Lim et al. 2017). This observation has been almost exclusively the result of an epidemic of papillary thyroid cancer (PTC), which now comprises approximately $84 \%$ of new cases (Lim et al. 2017). While radiation exposure, family history, and obesity are established risk factors, little research has investigated the role of other environmental exposures, which may be significant contributors to increasing PTC incidence (Kitahara and Sosa 2016).

Use of flame retardants (FRs) also increased over the last several decades due to the implementation of mandatory and voluntary flammability standards for furniture, electronics, and construction materials (Alaee et al. 2003; van der Veen and de Boer 2012). Polybrominated diphenyl ethers (PBDEs) were once among the most commonly used FRs in consumer products; they were routinely applied to furniture (Penta-BDE commercial mixture) and electronics (DecaBDE mixture). However, their persistence in the environment, high bio-accumulation potential, and possible toxicity led to their phase-out in many regions of the world beginning in the early2000s (Fromme et al.2016). Since that time, industry has turned to various alternatives to meet flammability standards, including alternate brominated FRs and organophosphate FRs (PFR) (Stapleton et al. 2012b; van der Veen and de Boer 2012).

These types of FRs are not chemically bound to the products in which they are used, leaving them predisposed to leach into the environment and resulting in widespread human exposure, particularly in home environments. They are ubiquitously detected in indoor dust 
samples, which is thought to be a primary source of exposure in the United States (e.g. (Lorber 2008; Stapleton et al. 2009; Watkins et al. 2013; Xu et al. 2016)); numerous studies have shown that levels of FRs in household dust are strongly correlated with biomarkers of exposure, and the Environmental Protection Agency estimates that $80 \%$ of the population's exposure to PBDE flame retardants is from indoor dust (Hoffman et al. 2014; Hoffman et al. 2015; Johnson et al. 2010; Lorber 2008; Stapleton et al. 2012a). Recent work suggests that although the levels of exposure to some FRs (e.g. Penta-BDE constituents) may be declining, human exposure to other FRs (e.g. PFRs) is likely increasing (Hoffman et al. 2017). This is particularly concerning, as emerging literature suggests that exposure to FRs is likely to impact human health (Allen et al. 2016; Meeker et al. 2013; Oulhote et al. 2016; Preston et al. 2017).

PBDEs share a similar chemical structure with thyroid hormones, and as such, they have received considerable attention with respect to their impact on thyroid regulation and clinically significant thyroid disease (Allen et al. 2016; Oulhote et al. 2016; Zhao et al. 2015). Although much less is known about the potential impact of other FRs, PFRs have been associated with alterations in thyroid hormone concentrations in some (Kim et al. 2015; Meeker and Stapleton 2010; Meeker et al. 2013; Preston et al. 2017; Wang et al. 2013; Xu et al. 2015) but not all studies (Moser et al. 2015).

Thyroid disease is associated with the growth of some cancers and has been linked to the prevalence of several types of cancer, including thyroid, suggesting that chemicals that disrupt thyroid hormone homeostasis in a significant way could contribute to cancer risk or severity (e.g. Lin et al. 2016; Hellevik et al. 2009; D'Avanzo et al. 2005; Brinton et al. 2007; Søgaard et al 2016; Moeller and Führer 2013). Given the relationship reported between FR exposures and thyroid hormone regulation, we hypothesize that exposure to FRs could increase cancer risk, and 
in particular thyroid cancer risk. Indeed, many FRs are considered carcinogens and have been

71 associated with the increased development of hepatocellular adenomas and carcinomas in

72 chronically exposed rodents. In separate studies, rats exposed to Deca-BDE and TCEP

73 experienced increased rates of thyroid gland follicular cell adenomas and carcinomas (NTP

$74 \quad$ 1991; NTP 1986).

Despite animal evidence indicating that the thyroid may be particularly sensitive to FRs, the impact of FR exposure on human thyroid cancer risk remains unknown, particularly for the

77 newer-use PFRs and alternative BFRs. To our knowledge, only one study has investigated this

78 potential association; Aschebrook-Kilfoy et al. (2015) reported no association between exposure

79 to Penta-BDEs and PTC (Aschebrook-Kilfoy et al. 2015), but other FRs, including BDE-209 and

80 the newer use FRs, were not investigated. Therefore, the current study was designed to test the

81 hypothesis that higher exposure to FRs in the home environment is associated with increased

82 odds of PTC. To accomplish this, a matched case-control study design was used. Traditional

83 biomarkers of PBDE exposure (i.e. serum PBDE levels) were employed; since suitable

84 biomarkers of long-term exposure do not exist for many other common FRs, relationships

85 between FRs in household dust and PTC also were evaluated. This represents the first study to

86 investigate relationships between PTC and many commonly used FRs detected in the home

87 environment.

\section{Subjects and Methods}

2.1 Study Participants

All study protocols were reviewed and approved by the Duke University Health System 
with PTC and referred to endocrinology or endocrine surgery at the Duke Cancer Institute or

Duke University Hospital were approached and invited to participate in the study by their treating physician. Willing participants then were contacted by our study team and enrolled. Control participants were recruited as described below and were matched to enrolled cases based on sex and age (within seven years of the cases' age at enrollment). Other Duke patients undergoing routine wellness care or care for unrelated medical issues were randomly selected and invited to participate as control participants. Flyers were placed in Duke University medical facilities as a means of recruiting additional control participants. Supplemental Figure 1 provides additional detail on participant recruitment and study component completion; for several matched pairs, only dust or blood samples were available for both the case and control. Paired blood and household dust samples were used for 92 participants, and other participants contributed either blood or household dust samples.

\subsubsection{Inclusion and exclusion criteria}

To reduce potential selection bias, inclusion was restricted to individuals living within 50 miles of Duke. To confirm that levels of exposure in the current home were reflective of exposure occurring over the last several years (e.g. before the diagnosis of PTC was established), inclusion was restricted to individuals that had lived in the same home for at least two years. Because a supplemental goal of our larger research effort was to evaluate the impact of FR exposure on thyroid function, pregnant women were excluded, as thyroid hormone levels vary considerably during pregnancy (Alemu et al. 2016). Inclusion of controls was restricted to individuals with no history of thyroid cancer or disease (current thyroid status was verified with biochemical testing). 
2.2. Clinical assessment

Clinical and pathologic information for the cases was obtained during a detailed review

119 of each PTC case's medical records, including the size of the primary tumor, focality of tumors within the thyroid gland (uni- or multi-focal), status of cervical lymph nodes (nodal metastases

present/absent) and distant metastases (present/absent), extra-thyroidal extension

123 (tumor/node/metastasis, or TNM) $7^{\text {th }}$ edition (Edge et al. 2010). These variables were generally 124 dichotomized for statistical analyses based on the distribution of data among cases. For example, 125 tumor size was classified as "small" for tumors less than $2 \mathrm{~cm}$ and "large" for tumors larger than 126 $2 \mathrm{~cm}$. In addition, BRAF V600E mutation status (+/-) was assessed for a subset of cases $(\mathrm{n}=45)$.

127 The BRAF V600E mutation (+) is common among PTCs and has been associated overall with more aggressive tumors; therefore, it may serve as an indicator of patient prognosis (Xing et al. 2013). Investigating relationships between exposure and BRAF mutations could provide 130 information about a potential mechanism by which FRs impact PTC occurrence.

\subsection{FRs in household dust}

Upon enrollment, study personnel visited each participant's home to obtain environmental

134 samples (e.g. household dust) and conduct study questionnaires. Participants were instructed not

135 to vacuum their home for at least two days prior to their study visit. During the visit, the main 136 living area of the home was vacuumed using a Eureka Mighty Might vacuum with a cellulose 137 thimble fitted in the hose attachment to collect the dust, similar to collection methods used in our 
previous studies (Stapleton et al. 2012a). Dust samples were wrapped in aluminum foil and immediately frozen upon collection.

Compounds assessed in dust included Penta-BDE constituents (i.e. BDE-47, BDE-99, BDE-100, BDE-153, and BDE-154), Deca-BDE (i.e. BDE-209), several commonly used PFRs [i.e. triphenyl phosphate (TPHP), tris(1,3-dichloroisopropyl)phosphate (TDCIPP), tris(1-chloro-2isopropyl)phosphate (TCIPP), and tris(2-chloroethyl) phosphate (TCEP)], and two alternative brominated flame retardants [2-ethylhexyl-2,3,4,5 tetrabromobenzoate (TBB or EH-TBB) and bis(2-ethylhexyl)-2,3,4,5-tetrabromophthalate (TBPH or BEH-TEBP)]. Dust samples were assessed for these compounds using previously published methods (Hoffman et al. 2015; Stapleton et al. 2012; Stapleton et al. 2014); briefly, samples (about $100 \mathrm{mg}$ ) were spiked with the following internal standards: d15-TDCIPP (154.8 ng), 13C-TPHP (100 ng), 13C-EH-TBB (100 ng), 13C-BEH-TEBP (100 ng), FBDE-69 (30.0 ng), and 13C BDE-209 (30.0 ng). The dust was extracted with 50:50 dichloromethane/hexane (v/v) via sonication extraction three times and then concentrated to $1.0 \mathrm{~mL}$ using a nitrogen evaporator system. These extracts were cleaned using Florisil solid-phase extraction (Supelclean ENVI-Florisil, 6 mL, 500 mg bed weight; Supelco), eluting the F1 fraction with $106 \mathrm{~mL}$ hexane (brominated compounds) and the F2 fraction with $10 \mathrm{~mL}$ ethyl acetate (PFRs). Each fraction was concentrated to about $1 \mathrm{~mL}$ and then transferred to an autosampler vial for analysis by GC/MS. Brominated flame retardants were quantified using GC/MS operated in electron capture negative ionization mode (GC/ECNI-MS), whereas the organophosphate flame retardants were quantified using GC/MS in electron impact mode (GC/EI-MS). Due to co-elution issues with BDE-99 and EH-TBB, extracts were also run on GC/EI-MS to quantify BDE-99 alone (monitoring the $[\mathrm{M}]+$ and $[\mathrm{M}-\mathrm{Br}]+$ fragments). Recovery of the internal standards was assessed using 13C-CDE141 for FBDE-69 and 13C 
BDE-209, d9-tris(2-chloroethyl) phosphate (d9-TCEP; $227 \mathrm{ng}$ ) for d15-TDCIPP, and d15triphenyl phosphate (d15-TPHP; $128 \mathrm{ng}$ ) for 13C-TPHP. Recoveries of FBDE-69, 13C-BDE209, d15-TDCIPP, and 13C-TPHP were on average 75, 70, 97, and 106\%, respectively. Standard Reference Material (SRM) 2585 (National Institute of Standards \& Technology, Gaithersburg, MD) was used to ensure accuracy and ranged from 73 to $111 \%$ relative to the certified values.

\subsection{Serum PBDEs}

All study participants were asked to provide non-fasting blood samples in which PBDEs were measured. Serum samples were assessed for 27 PBDEs as described in Butt et al. 2016. Briefly, serum samples were spiked with $2.5 \mathrm{ng}$ of FBDE-69. Samples were sonicated with 2.0 $\mathrm{mL}$ 0.1 $\mathrm{M}$ formic acid and $6.0 \mathrm{~mL}$ water to denature serum proteins. Following conditioning of the column with $5.0 \mathrm{~mL}$ dichloromethane, methanol, and water each, the samples were loaded on a Waters Oasis HLB column (500 mg bed weight, $6 \mathrm{~mL}$ ) and washed with $5.0 \mathrm{~mL}$ water. PBDE analytes were eluted with $10.0 \mathrm{~mL}$ of $1: 1$ dichloromethane/ethyl acetate $(\mathrm{v} / \mathrm{v})$ then concentrated to near dryness using a nitrogen evaporator and reconstituted in $1.0 \mathrm{~mL}$ hexane. These samples were further cleaned using a silica column cartridge ( $1 \mathrm{~g}$, Waters, Sep-Pak), eluting the F1 fraction with $10.0 \mathrm{~mL}$ hexane for the PBDEs. The F1 fraction was concentrated to about $100 \mu \mathrm{L}$ and spiked with $5.0 \mathrm{ng}$ 13C-CDE-141 to assess recovery of FBDE-69 and 13C-BDE-209, respectively. This fraction was analyzed using GC/MS in electron capture negative ionization mode for twenty-seven PBDEs. Recoveries of FBDE-69 averaged 67\%. Standard Reference Material (SRM) 1958 (National Institute of Standards \& Technology, Gaithersburg, MD) was used to ensure accuracy. Measurements in SRM 1958 relative to the certified values were $129 \%$ 
for BDE-47 and 75\% for BDE-153.Statistical analyses were conducted for congeners detected in greater than $70 \%$ of serum samples. Because PBDEs can bind to lipids in serum, individual BDE measures were lipid-corrected prior to statistical analysis using measurements of total cholesterol and triglycerides (Covaci et al. 2006). Lipid measurement was conducted by LabCorp in Burlington, NC using standard protocols. However, analyses were also conducted with wet weight PBDE concentrations, and nearly identical results were obtained. To facilitate comparisons with other studies, we present serum concentrations and results from lipid corrected analyses.

\subsection{Statistical analyses}

Descriptive statistics were calculated to examine the detection and distribution of FRs in serum and household dust. Concentrations were log-normally distributed, and preliminary analyses suggested that associations between FRs and outcomes were unlikely to be linear. As such, non-parametric statistical analyses were used or levels of each FR were dichotomized at the median value among controls to represent 'high' and 'low' exposure in predictive models. Kruskal-Wallis tests were used to assess bivariate associations between FRs and PTC outcomes. Logistic regression models were used to examine associations between exposure and case status while controlling for potential confounding factors. Standard polytomous regression (i.e. multinomial regression) analyses were used to evaluate relationships between exposure and outcomes with multiple levels (tumor size, histopathology, etc.).

Regression analyses were adjusted for participant age and household income, which are variables hypothesized to be related to both FR exposures and thyroid cancer risk or diagnosis. Ten participants chose not to provide their household income; for these participants, income was 
207 imputed as the average household income in the census tract in which they were living at the

208 time of enrollment. Analyses were conducted including body mass index (BMI) as a covariate

209 (categorical as in Table 1). However, it is possible that BMI may be on the causal pathway

210 between FR exposure and PTC; therefore, analyses also were performed that excluded BMI.

211 Results were nearly identical; thus, BMI-adjusted models are presented. In addition to these

212 variables, race (white vs. non-white) and employment status (employed vs. unemployed) were

213 considered to be potential confounders, but neither impacted effect estimates. Additionally,

214 confounding by cigarette smoking was considered; current smoking was uncommon among

215 participants ( $\mathrm{n}=7$ current smokers), and therefore it was not included in analyses. Participants

216 were also asked about their past exposure to ionizing radiation. None reported significant prior

217 exposure, and accordingly, radiation was not included in analyses.

218 Because FRs are highly correlated in both dust and serum (making it difficult to include

219 multiple exposures in a single model), separate models were constructed for each FR while

220 recognizing that exposures do not occur in isolation. Accordingly, principle component analyses

221 were conducted to systematically assess FR mixtures. Results of these analyses did not provide

222 any additional insights and are not shown.

223 All statistical analyses were conducted using SAS version 9.4 (SAS Institute, Cary, North

224 Carolina). Statistical significance was set at a $\mathrm{p}<0.05$ and we did not perform adjustment for

225 multiple comparisons, as has been recommended in the epidemiologic literature (Rothman

226 1990).

227 3. Results

228

3.1. Study population 
Reflecting known gender differences in PTC risk, our final study population was $78.6 \%$ female (Table 1). The mean age of study participants was 48 years (among cases, 39\% were $<45$ years of age, a threshold used in AJCC staging (Edge et al. 2010)). Cases and controls were similar with respect to race and ethnicity, household income, and health history. Cases and controls were also similar with respect to the number of years they reported living at the current address, which was more than 10 years for both. Among PTC cases, the majority were AJCC stage $1(62.9 \%)$, and tumors were generally contained to the thyroid (70\%; Table 1$)$. The BRAF V600E mutation was common; $62.2 \%$ of the 45 cases with BRAF V600E assessment were positive for the mutation.

\subsection{FRs in household dust}

FRs were detected in all house dust samples, and concentrations spanned several orders of magnitude, similar to other studies in the United States (Dodson et al. 2012; Hoffman et al. 2014; K. Hoffman et al. 2015; Stapleton et al. 2008; Stapleton et al. 2012a). As a chemical class, PFRs were detected most frequently and in the highest concentrations (Figure 1). For example, median TCIPP concentrations in household dust were over $2000 \mathrm{ng} / \mathrm{g}$ (i.e. parts per billion) for both cases and controls, similar to what has been reported in the literature. As is frequently observed, FRs in household dust were correlated (Supplemental Table 1). The highest correlations were observed between PBDE congeners used in the PentaBDE FR mixture $\left(r_{s}=0.70-0.88\right)$ and between TBB and TBPH, which are both used in Firemaster ${ }^{\circledR} 550$, a commonly applied flame retardant mixture. Bivariate analyses demonstrated significant (i.e. BDE $209 \mathrm{p}=0.05$ ), or near significant (i.e. TCEP $\mathrm{p}=0.13$ and TPHP $\mathrm{p}=0.12$ ) differences in the median dust FR concentrations in the homes of cases and controls. After adjustment for potential 
252 253

confounding by participant, household income, and body mass index, PTC cases were significantly more likely to have high concentrations of TCEP and BDE-209 in their house dust (Table 2). For example, those with dust BDE-209 levels above the median were 2.29 times as likely to be cases compared to those with house dust levels below the median (95\% Confidence Interval $[\mathrm{CI}]: 1.03,5.08, \mathrm{p}=0.04)$. In addition, results were suggestive of an association between higher dust TPHP concentrations and PTC, but these did not reach statistical significance (odds ratio $(\mathrm{OR})=2.07 ; 95 \%$ CI: $0.94,4.56 ; \mathrm{p}=0.07)$. The levels of other FRs in household dust were not associated with the odds of PTC in bivariate or multivariate analyses.

\subsection{Dust FRs and measures of tumor aggressiveness}

FRs also were associated with markers of tumor aggressiveness (Table 3; results not shown for bivariate analyses). For example, high levels of BDE-209 were only associated with tumors contained in the thyroid, those that were $\mathrm{pT} 1 \mathrm{a}$ or $\mathrm{pT} 1 \mathrm{~b}$ and $\mathrm{pN} 0$ (low stage indicating tumor is less than $2 \mathrm{~cm}$ and has not spread to the lymph nodes), suggesting that BDE-209 may contribute to the risk of smaller, less aggressive PTCs. Associations between TPHP and PTC also were stronger for pT1a and $\mathrm{pT} 1 \mathrm{~b}$ tumors (tumors less than $2 \mathrm{~cm}$ ). Conversely, higher levels of TCEP were associated with extrathyroidal extension, more advanced T-stage, and nodal metastasis. Of note, AJCC stage was considered as a potential outcome. Results tended to suggest that BDE-209, TPHP, and TCEP all were associated with higher AJCC stage, potentially as an artifact of residual confounding by age, which is inherently related to AJCC staging and which was associated with FRs in this study. AJCC stage results are shown in Supplemental Table 2. 


\subsection{Dust FRs and BRAF V600E mutation}

Associations between FRs and PTC varied by the presence of the BRAF V600E mutation, with high exposure generally more strongly related to BRAF V600E(-) tumors (Table 4). For example, in adjusted analyses, participants with high levels of BDE-209 in house dust were 14.2 times as likely to be BRAF(-) cases compared to controls, although confidence intervals were quite wide (95\% CI: $1.63,123 ; \mathrm{p}=0.02)$. Although other cases also were more likely to have high levels of BDE-209 in their homes, associations were not statistically significant for BRAF(+) cases or participants for whom BRAF was not assessed. A similar pattern was observed for TPHP, with stronger associations between exposure and BRAF(-) tumors. Although TCEP followed a similar pattern, with BRAF V600E(-) cancers more strongly linked with high exposure, results were not statistically significant in analysis where case status was stratified by the presence or absence of the BRAF V600E mutation.

\subsection{Serum PBDE FRs}

Of the 27 PBDEs measured in serum samples, only two were detected in more than $70 \%$ of serum samples, and spanning several orders of magnitude, similar to other studies (Sjodin et al. 2008). The median concentrations of BDE-47 and BDE-153 in serum were 9.9 and $5.0 \mathrm{ng} / \mathrm{g}$ lipid among controls, respectively, and 8.9 and $4.1 \mathrm{ng} / \mathrm{g}$ lipid among cases ( $\mathrm{p}>0.05)$. BDE-47 in serum was significantly correlated with BDE-47 in dust $\left(r_{s}=0.35, p=0.004\right)$, but no relationship between BDE-153 in dust and serum was observed. There was no evidence of association between serum BDE-47 and BDE-153 levels and PTC (Supplemental Table 2). Investigating more-detailed case definitions (e.g. presence of BRAF V600E mutation) did not provide additional insight (Supplemental Table 3). 


\section{Discussion}

The incidence of PTC has increased over the past several decades, a period over which

the use of FRs also increased. Our results from this case-control study support our original

hypothesis and suggest that exposure to some FRs in the home environment (i.e. BDE-209 and TCEP) may be related to increased risk for the development of clinically significant PTC. To our knowledge, this is the first work to assess associations between PTC and exposure to PFRs, alternate BFRs, and Deca-BDE. In addition, this work investigated associations based on genetics/mutation status, which is a major strength of our study, and highlights a need to further investigate environment and gene interactions in cancer research. We observed the strongest association for Deca-BDE and BRAF negative tumors, suggesting an alternate mutation or mechanistic pathway between exposure and PTC.

While thyroid cancers of all sizes have been observed to increase in the United States

311 over the last 30 years, smaller PTCs $(<2 \mathrm{~cm}$, and especially $\leq 1 \mathrm{~cm})$ appear to have increased at 312 the fastest rate (Chen et al. 2009). Chen et al. reported that between 1988 and 2005, incidence 313 rates for tumors $<1.0 \mathrm{~cm}$ increased by an average of 8.6 percent annually, while the incidence of tumors $\geq 4 \mathrm{~cm}$ increased at 5.7 percent per year (among women) (Chen et al. 2009). Many have suggested that this could be the result of surveillance bias based on increasing use of diagnostic 316 imaging like ultrasound, CT, MRI, and PET scanning, resulting in the finding of more 317 'incidental' thyroid nodules that represent thyroid cancers that are subclinical (Chen et al. 2009; 318 Ho et al. 2015; Kitahara and Sosa 2016). While there are certainly other potential explanations 319 for observed incidence trends, our results suggest that exposure to BDE-209 in house dust may 320 be associated with an increased risk for the development of these small PTCs. A similar pattern 
was observed for TPHP, although this finding was not statistically significant. If BDE-209 is playing a significant role in the etiology of small PTCs, the incidence rate of these types of tumors would be expected to begin to decline in the coming decades due to the voluntary DecaBDE phase-out. However, our results suggest that TPHP also might contribute to an increased risk for small PTCs, and data suggest that exposure to TPHP may have increased over the last decade (Hoffman et al. 2017). Perhaps of more concern, our results suggest that exposure to TCEP may be associated with increased risk of more aggressive PTCs.

A previous study investigated associations between serum biomarkers of Penta-BDEs and thyroid cancer, finding no associations (Aschebrook-Kilfoy et al. 2015). Similar to previous work, associations between Penta-BDE compounds in serum or household dust were not associated with PTC in our present work. However, other FRs, including BDE-209, were not investigated in the work of Aschebrook-Kilfoy et al. (2015). Using house dust as a measure of long-term chronic exposure may have many benefits over traditional approaches of using serum biomarkers, particularly as it allows for the measurement and detection of a wider range of FRs.

Our results should be interpreted in the context of several important limitations. Analyses largely relied on the levels of FRs in the home environment as a proxy for personal exposure. While this approach is likely to result in some misclassification because exposure in other environments is not captured (e.g. at work or in the car), it is an efficient means of assessing exposure to a wide range of FRs that occur in mixtures, particularly those for which long-term exposure biomarkers have not been validated. PFRs, for example, are rapidly metabolized and excreted in urine; therefore, assessing urinary concentrations at the time of diagnosis may not be reflective of past average/chronic exposures. Nonetheless, because sources of exposure to PFRs may be relatively constant over time, the collection of urine samples in 
344 future studies could provide additional insights. Unfortunately, urine samples were not collected

345 for the majority of participants in our current work. Household dust FR concentrations are

346 thought to be correlated over the course of several years (Stapleton et al. 2014; Whitehead et al.

347 2013; Dodson et al. 2012) and are highly correlated with personal exposure (Bramwell 2016;

348 Hoffman et al. 2014; Hoffman et al. 2015; Stapleton et al. 2012a). To ensure that dust

349 measurements were reflective of exposure preceding diagnosis, study participation was restricted

350 to individuals that had lived in their homes for a minimum of two years. However, participants

351 lived in their homes for an average of more than10 years at the time of enrollment, suggesting

352 that FR measures in dust likely reflect longer-term exposures, although we acknowledge that the

353 latency period between exposure to FRs and the development of PTC could be substantially

354 longer. A cohort study design with long-term follow-up would be better suited to address this

355 issue; however, we are unaware of any cohort studies that are banking novel exposure markers,

356 such as household dust. In addition, exposures to flame retardants occur in mixtures. Although

357 we considered the use of PCA to assess the impacts of commonly occurring FR mixtures, our

358 sample size limited our ability to assess the joint impact of multiple FRs simultaneously.

359 Additionally assessing mixtures of FRs should be included as a goal of future studies with a

360 sufficient sample size.

$361 \quad$ 5. Conclusion

362 With the incidence of thyroid cancer quickly increasing and little knowledge of what may be

363 leading to this drastic increase (outside of 'over diagnosis'), understanding potential

364 environmental factors contributing to thyroid cancer is critical. Our results suggest that exposure

365 to BDE-209 and TCEP in the home environment may be associated with an increased risk of

366 PTC. This is a critical concern, particularly as the use of FRs is expected to increase in the future 
367 (Green 2015). Given the increase in mortality associated with PTC (Lim et al. 2017) and the 368 high financial demands placed upon thyroid patients for treatment and follow-up, more research 369 is urgently needed to investigate these associations and determine if these trends are replicated in 370 a larger cohort. 


\section{References}

Alaee M, Arias P, Sjodin A, Bergman A. 2003. An overview of commercially used brominated flame retardants, their applications, their use patterns in different countries/regions and possible modes of release. Environ Int 29:683-689.

Alemu A, Terefe B, Abebe M, Biadgo B. 2016. Thyroid hormone dysfunction during pregnancy: A review. Int J Reprod Biomed (Yazd) 14:677-686.

Allen JG, Gale S, Zoeller RT, Spengler JD, Birnbaum L, McNeely E. 2016. Pbde flame retardants, thyroid disease, and menopausal status in us women. Environ Health-Glob 15.

Aschebrook-Kilfoy B, DellaValle CT, Purdue M, Kim C, Zhang Y, Sjodin A, et al. 2015. Polybrominated diphenyl ethers and thyroid cancer risk in the prostate, colorectal, lung, and ovarian cancer screening trial cohort. Am J Epidemiol 181:883-888.

Bramwell L GS, Rankin J, et al. 2016. Associations between human exposure to polybrominated diphenyl ether flame retardants via diet and indoor dust, and internal dose: A systematic review. Environ Int 92-93:680-694.

Brinton LA, Sakoda LC, Frederiksen K, Sherman ME, Kjaer SK, Graubard BI, et al. 2007. Relationships of uterine and ovarian tumors to pre-existing chronic conditions. Gynecol Oncol 107:487-494.

Butt CM, Miranda ML, Stapleton HM. 2016. Development of an analytical method to quantify PBDEs, OH-BDEs, HBCDs, 2,4,6-TBP, EH-TBB, and BEH-TEPH in human serum. Anal Bioanal Chem 408:2449-2459.

Chen AY, Jemal A, Ward EM. 2009. Increasing incidence of differentiated thyroid cancer in the united states, 1988-2005. Cancer 115:3801-3807.

Covaci A, Voorspoels S, Thomsen C, van Bavel B, Neels H. 2006. Evaluation of total lipids using enzymatic methods for the normalization of persistent organic pollutant levels in serum. Sci Total Environ 366:361-366.

D'Avanzo B, La Vecchia C, Franceschi S, Negri E, Talamini R. 1995. History of thyroid diseases and subsequent thyroid cancer risk. Cancer Epidemiol Biomarkers Prev 4:193-199.

Dodson RE, Perovich LJ, Covaci A, Van den Eede N, Ionas AC, Dirtu AC, et al. 2012. After the pbde phase-out: A broad suite of flame retardants in repeat house dust samples from california. Environ Sci Technol 46:13056-13066.

Edge S, Byrd D, Compton C, Fritz A, Greene F, Trotti A, eds. 2010. AJCC cancer staging manual (7th ed). New York, NY.

Fromme H, Becher G, Hilger B, Volkel W. 2016. Brominated flame retardants - exposure and risk assessment for the general population. Int J Hyg Environ Health 219:1-23. 
Green M. 2015. Flame retardant chemicals: Technologies and global markets. Wellesley, MA:BCC Research.

Hellevik AI, Asvold BO, Bjoro T, Romundstad PR, Nilsen TI, Vatten LJ. 2009. Thyroid function and cancer risk: A prospective population study. Cancer Epidemiol Biomarkers Prev 18:570-574.

Ho AS, Davies L, Nixon IJ, Palmer FL, Wang LY, Patel SG, et al. 2015. Increasing diagnosis of subclinical thyroid cancers leads to spurious improvements in survival rates. Cancer 121:17931799.

Hoffman K, Fang M, Horman B, Patisaul HB, Garantziotis S, Birnbaum LS, et al. 2014. Urinary tetrabromobenzoic acid (TBBA) as a biomarker of exposure to the flame retardant mixture Firemaster ${ }^{\circledR}$ 550. Environ Health Perspect 122:963-969.

Hoffman K, Garantziotis S, Birnbaum LS, Stapleton HM. 2015. Monitoring indoor exposure to organophosphate flame retardants: Hand wipes and house dust. Environ Health Perspect 123:160-165.

Hoffman K, Butt C, Webster T, Preston E, Hammel S, Makey C, et al. 2017. Temporal trends in exposure to organophophate flame retardants in the united states. Environ Sci Technol Let Ahead of Print.

Johnson PI, Stapleton HM, Sjodin A, Meeker JD. 2010. Relationships between polybrominated diphenyl ether concentrations in house dust and serum. Environ Sci Technol 44:5627-5632.

Kim S, Jung J, Lee I, Jung D, Youn H, Choi K. 2015. Thyroid disruption by triphenyl phosphate, an organophosphate flame retardant, in zebrafish (danio rerio) embryos/larvae, and in gh3 and frtl-5 cell lines. Aquat Toxicol 160:188-196.

Kitahara CM, Sosa JA. 2016. The changing incidence of thyroid cancer. Nat Rev Endocrinol 12:646-653.

Kuijpens JLP, Nyklictek I, Louwman MWJ, Weetman TAP, Pop VJM, Coebergh JWW 2005. Hypothyroidism might be related to breast cancer in post-menopausal women. Thyroid 15:12531259.

Lim H, Devesa SS, Sosa JA, Check D, Kitahara CM. 2017. Trends in thyroid cancer incidence and mortality in the united states, 1974-2013. JAMA 317(13):1338-1348.

Lin HY, Chin YT, Yang YC, Lai HY, Wang-Peng J, Liu LF, et al. 2016. Thyroid hormone, cancer, and apoptosis. Compr Physiol 6:1221-1237.

Lorber M. 2008. Exposure of americans to polybrominated diphenyl ethers. J Expo Sci Environ Epidemiol 18:2-19.

Meeker JD, Stapleton HM. 2010. House dust concentrations of organophosphate flame retardants in relation to hormone levels and semen quality parameters. Environ Health Perspect 118:318323. 
Meeker JD, Cooper EM, Stapleton HM, Hauser R. 2013. Exploratory analysis of urinary metabolites of phosphorus-containing flame retardants in relation to markers of male reproductive health. Endocr Disruptors (Austin) 1:e26306.

Moeller LC, Fuhrer D. 2013. Thyroid hormone, thyroid hormone receptors, and cancer: A clinical perspective. Endocr Relat Cancer 20:R19-29.

Moser VC, Phillips PM, Hedge JM, McDaniel KL. 2015. Neurotoxicological and thyroid evaluations of rats developmentally exposed to tris(1,3-dichloro-2-propyl)phosphate (TDCIPP) and tris(2-chloro-2-ethyl)phosphate (TCEP). Neurotoxicol Teratol. 52(Pt B):236-47. NTP (National Toxicology Program). 1991. NTP toxicology and carcinogenesis studies of tris(2chloroethyl) phosphate (cas no. 115-96-8) in $\mathrm{f} 344 / \mathrm{n}$ rats and b6c3f1 mice (gavage studies). Natl Toxicol Program Tech Rep Ser 391:1-233.

NTP (National Toxicology Program). 1986. Toxicology and carcinogenesis studies of

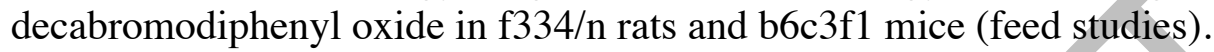

Oulhote Y, Chevrier J, Bouchard MF. 2016. Exposure to polybrominated diphenyl ethers (PBDEs) and hypothyroidism in canadian women. J Clin Endocrinol Metab 101:590-598.

Preston EV, McClean MD, Henn BC, Stapleton HM, Braverman LE, Pearce EN, et al. 2017. Associations between urinary diphenyl phosphate and thyroid function. Environ Int. 101; 158164.

Rothman KJ. 1990. No adjustments are needed for multiple comparisons. Epidemiology 1:4346.

Sjodin A, Wong LY, Jones RS, Park A, Zhang Y, Hodge C, et al. 2008. Serum concentrations of polybrominated diphenyl ethers (PBDEs) and polybrominated biphenyl (PBB) in the United States population: 2003-2004. Environ Sci Technol 42:1377-1384.

Stapleton HM, Allen JG, Kelly SM, Konstantinov A, Klosterhaus S, Watkins D, et al. 2008. Alternate and new brominated flame retardants detected in U.S. House dust. Environ Sci Technol 42:6910-6916.

Stapleton HM, Klosterhaus S, Eagle S, Fuh J, Meeker JD, Blum A, et al. 2009. Detection of organophosphate flame retardants in furniture foam and u.S. House dust. Environ Sci Technol 43:7490-7495.

Stapleton HM, Eagle S, Sjodin A, Webster TF. 2012a. Serum pbdes in a north carolina toddler cohort: Associations with handwipes, house dust, and socioeconomic variables. Environ Health Perspect 120:1049-1054.

Stapleton HM, Eagle S, Sjoedin A, Webster TF. 2012. Serum pbdes in a north carolina toddler cohort: Associations with handwipes, house dust, and socioeconomic variables. Environ Health Perspect 120:1049-1054. 
Stapleton HM, Sharma S, Getzinger G, Ferguson PL, Gabriel M, Webster TF, et al. 2012b. Novel and high volume use flame retardants in us couches reflective of the 2005 pentabde phase out. Environ Sci Technol 46:13432-13439.

Stapleton HM, Misenheimer J, Hoffman K, Webster TF. 2014. Flame retardant associations between children's handwipes and house dust. Chemosphere 116:54-60.

van der Veen I, de Boer J. 2012. Phosphorus flame retardants: Properties, production, environmental occurrence, toxicity and analysis. Chemosphere 88:1119-1153.

Wang Q, Liang K, Liu J, Yang L, Guo Y, Liu C, et al. 2013. Exposure of zebrafish embryos/larvae to tdcpp alters concentrations of thyroid hormones and transcriptions of genes involved in the hypothalamic-pituitary-thyroid axis. Aquat Toxicol 126:207-213.

Watkins DJ, McClean MD, Fraser AJ, Weinberg J, Stapleton HM, Webster TF. 2013. Associations between PBDEs in office air, dust, and surface wipes. Environ Int 59:124-132.

Whitehead TP, Brown FR, Metayer C, Park J-S, Does M, Petreas MX, et al. 2013. Polybrominated diphenyl ethers in residential dust: Sources of variability. Environ Int 57-58:1124.

Xing M, Alzahrani AS, Carson KA, Viola D, Elisei R, Bendlova B, et al. 2013. Association between BRAF V600E mutation and mortality in patients with papillary thyroid cancer. JAMA 309:1493-1501.

Xu F, Giovanoulis G, van Waes S, Padilla-Sanchez JA, Papadopoulou E, Magner J, et al. 2016. Comprehensive study of human external exposure to organophosphate flame retardants via air, dust, and hand wipes: The importance of sampling and assessment strategy. Environ Sci Technol 50:7752-7760.

Xu T, Wang Q, Shi Q, Fang Q, Guo Y, Zhou B. 2015. Bioconcentration, metabolism and alterations of thyroid hormones of tris(1,3-dichloro-2-propyl) phosphate (TDCPP) in zebrafish. Environ Toxicol Pharmacol 40:581-586.

Zhao XM, Wang HL, Li J, Shan ZY, Teng WP, Teng XC. 2015. The correlation between polybrominated diphenyl ethers (PBDEs) and thyroid hormones in the general population: A meta-analysis. Plos One 10. 
Table 1: Selected demographic and pathologic characteristics of 140 study participants.

\begin{tabular}{|c|c|c|c|c|c|}
\hline & \multicolumn{2}{|c|}{ Cases $(n=70)$} & \multicolumn{2}{|c|}{ Controls $(n=70)$} & \multirow[b]{2}{*}{ p-value } \\
\hline & $\begin{array}{c}\text { Mean } \pm \text { St. Dev. } \\
\text { or } n\end{array}$ & $\begin{array}{l}\text { Range } \\
\text { or } \%\end{array}$ & $\begin{array}{c}\text { Mean } \pm \text { St. Dev. } \\
\text { or } n\end{array}$ & $\begin{array}{l}\text { Range } \\
\text { or \% }\end{array}$ & \\
\hline Age (years) & $48.6 \pm 11.8$ & $26-75$ & $48.1 \pm 11.8$ & $28-80$ & 0.8 \\
\hline Years in Current Home ${ }^{\text {a }}$ & $11.5 \pm 10.8$ & $2-69$ & $10.9 \pm 9.6$ & $2-46$ & 0.75 \\
\hline \multicolumn{6}{|l|}{ Sex } \\
\hline Male & 15 & 21.4 & 15 & 21.4 & -- \\
\hline Female & 55 & 78.6 & 55 & 78.6 & \\
\hline \multicolumn{6}{|l|}{ Race } \\
\hline White & 54 & 77.1 & 56 & 80.0 & \\
\hline African American & 10 & 14.3 & 11 & 15.7 & \\
\hline Other & 6 & 8.6 & 3 & 4.3 & \\
\hline \multicolumn{6}{|l|}{ Ethnicity } \\
\hline Non-Hispanic or Latino & 66 & 94.3 & 69 & 98.6 & 0.21 \\
\hline Hispanic or Latino & 4 & 5.7 & 1 & 1.4 & \\
\hline \multicolumn{6}{|l|}{ Annual Household Income ${ }^{\text {b }}$} \\
\hline$<\$ 50,000$ & 16 & 22.9 & 14 & 20.0 & 0.59 \\
\hline$\$ 50-100,000$ & 24 & 34.3 & 20 & 28.6 & \\
\hline$>\$ 100,000$ & 30 & 42.9 & 36 & 51.4 & \\
\hline History of Other Cancer & 11 & 15.7 & 10 & 14.3 & 0.75 \\
\hline \multicolumn{6}{|l|}{ BMI } \\
\hline Underweight or normal & 42 & 60.0 & 37 & 52.9 & 0.39 \\
\hline Overweight or obese & 28 & 40.0 & 33 & 47.1 & \\
\hline \multicolumn{6}{|l|}{ AJCC stage } \\
\hline 1 & 44 & 62.9 & -- & -- & -- \\
\hline 2,3 , or 4 & 23 & 32.9 & -- & -- & \\
\hline \multirow{2}{*}{\multicolumn{6}{|c|}{ T-Stage }} \\
\hline & & & & & -- \\
\hline 2,3 , or 4 & 31 & 44.3 & -- & -- & \\
\hline & 3 & 4.3 & -- & -- & \\
\hline N-Stage & 31 & 44.3 & -- & -- & -- \\
\hline $1 \mathrm{a}$ or $1 \mathrm{~b}$ & 23 & 32.9 & -- & -- & \\
\hline & 13 & 18.6 & -- & -- & \\
\hline & 3 & 4.3 & -- & -- & \\
\hline \multicolumn{6}{|l|}{ BRAF V600E } \\
\hline$(+)$ & 28 & 40.0 & -- & -- & -- \\
\hline$(-)$ & 17 & 24.3 & -- & -- & \\
\hline not assessed ${ }^{\mathrm{d}}$ & 25 & 35.7 & -- & -- & \\
\hline \multicolumn{6}{|l|}{ Extrathyroidal extension } \\
\hline Present & 17 & 24.3 & -- & -- & -- \\
\hline Absent & 49 & 70.0 & -- & -- & \\
\hline not available ${ }^{\mathrm{c}}$ & 4 & 5.7 & -- & -- & \\
\hline
\end{tabular}

${ }^{\mathrm{a}}$ Residential duration information was missing for 7 participants. As a requirement for enrollment, it was verified that all participants had lived in their current home at least 2 years.

$508{ }^{b} 10$ participants chose not to provide income information. For these participants, income was imputed as the median 509 household income for their census tract.

510 c NA-not available, because the patient did not have surgery at Duke or information was not included in their Duke 511 medical record.

$512{ }^{\mathrm{d}}$ BRAF V600E status was assessed for a subset of participants. 
Table 2: Adjusted odds ratios for PTC for FR exposure above the median $(n=116)$.

\begin{tabular}{lcc}
\hline Mixture & Individual FR & OR $(\mathbf{9 5 \%}$ CI), p-value \\
\hline Alternate BFRs & TBB & $0.62(0.29,1.31), \mathrm{p}=0.21$ \\
& TBPH & $1.22(0.56,2.65), \mathrm{p}=0.61$ \\
& & \\
PFRs & TPHP & $2.07(0.94,4.56), \mathrm{p}=0.07$ \\
& TDCIPP & $1.49(0.69,3.20), \mathrm{p}=0.31$ \\
& TCEP & $2.42(1.10,5.33), \mathrm{p}=0.03$ \\
& TCIPP & $0.92(0.43,1.97), \mathrm{p}=0.83$ \\
& & \\
Penta-BDE & BDE-47 & $0.80(0.38,1.70), \mathrm{p}=0.57$ \\
& BDE-99 & $0.75(0.36,1.59), \mathrm{p}=0.45$ \\
& BDE-100 & $0.88(0.42,1.87), \mathrm{p}=0.74$ \\
& BDE-153 & $0.77(0.37,1.63), \mathrm{p}=0.50$ \\
& BDE-154 & $0.80(0.38,1.70), \mathrm{p}=0.56$ \\
Deca-BDE & BDE-209 & $2.29(1.03,5.08), \mathrm{p}=0.04$ \\
\hline
\end{tabular}

515

516

517

518

519

520

521

522

523

524

525

526

527

528

529

530

531

532

533 
Table 3: Adjusted odds ratios by indicator of tumor aggressiveness for FR exposure above the median ( $n=108$ for extra thyroidal extension and $n=110$ for T-stage and N-stage).

\section{Extra-thyroidal extension}

FR

TBB

Present OR $(95 \%$ CI $)$
T-stage

Stage OR (95\% CI)
N-stage

Stage OR $(95 \%$ CI $)$

$\begin{array}{cccc}\text { No } & 0.72(0.31,1.64) & \text { 1a or } 1 \mathrm{~b} & 0.52(0.20,1.34) \\ \text { Yes } & 0.49(0.14,1.66) & 2,3 \text { or } 4 & 0.8(0.31,2.09) \\ & & & \\ \text { No } & 1.28(0.55,3.00) & 1 \text { a or } 1 \mathrm{~b} & 1.29(0.50,3.31) \\ \text { Yes } & 1.01(0.31,3.30) & 2,3 \text { or } 4 & 1.11(0.42,2.96)\end{array}$

$0.44(0.10,1.94)$

$0 \quad 0.59(0.21,1.60)$

$1 \quad 0.79(0.28,2.21)$

\begin{tabular}{|c|c|c|c|c|c|c|}
\hline \multirow[t]{3}{*}{ TBPH } & No & $1.28(0.55,3.00)$ & $1 a$ or $1 b$ & $1.29(0.50,3.31)$ & $\mathrm{X}$ & $0.61(0.15,2.58)$ \\
\hline & Yes & $1.01(0.31,3.30)$ & 2,3 or 4 & $1.11(0.42,2.96)$ & 0 & $0.97(0.36,2.66)$ \\
\hline & & & & & 1 & $2.04(0.68,6.18)$ \\
\hline \multirow[t]{2}{*}{ ТPHP } & No & $2.11(0.88,5.04)$ & $1 \mathrm{a}$ or $1 \mathrm{~b}$ & $3.63(1.26,10.4)^{*}$ & $\mathrm{X}$ & $4.81(0.86,27.0)$ \\
\hline & Yes & $2.03(0.59,6.99)$ & 2,3 or 4 & $1.23(0.46,3.27)$ & & $1.91(0.68,5.39)$ \\
\hline \multirow[t]{3}{*}{ TDCIPP } & No & $1.33(0.57,3.13)$ & $1 \mathrm{a}$ or $1 \mathrm{~b}$ & $1.43(0.55,3.68)$ & $\bar{X}$ & $0.82(0.19,3.5)$ \\
\hline & Yes & $2.74(0.76,9.87)$ & 2,3 or 4 & $1.81(0.66,4.95)$ & 0 & $1.61(0.58,4.53)$ \\
\hline & & & & & 1 & $2.16(0.72,6.48)$ \\
\hline \multirow[t]{3}{*}{ TCEP } & No & $2.13(0.89,5.07)$ & $1 \mathrm{a}$ or $1 \mathrm{~b}$ & $2.07(0.79,5.44)$ & $\mathrm{X}$ & $9.70(1.09,86.2)^{*}$ \\
\hline & Yes & $4.14(1.01,17.0)^{*}$ & 2,3 or 4 & $3.18(1.08,9.38)^{*}$ & 0 & $1.23(0.45,3.37)$ \\
\hline & & & & & 1 & $4.06(1.18,13.9)^{*}$ \\
\hline \multirow[t]{3}{*}{ TCIPP } & No & $0.95(0.41,2.21)$ & $1 \mathrm{a}$ or $1 \mathrm{~b}$ & $1.19(0.47,3.02)$ & $\mathrm{X}$ & $0.82(0.2,3.42)$ \\
\hline & Yes & $1.10(0.33,3.65)$ & 2,3 or 4 & $0.78(0.29,2.10)$ & 0 & $1.05(0.38,2.87)$ \\
\hline & & & & & 1 & $0.95(0.33,2.70)$ \\
\hline \multirow[t]{3}{*}{ BDE-47 } & No & $0.69(0.30,1.60)$ & 1a & $0.85(0.34,2.15)$ & $\mathrm{X}$ & $0.61(0.15,2.55)$ \\
\hline & Yes & $0.96(0.29,3.13)$ & 2,3 or 4 & $0.65(0.24,1.72)$ & 0 & $0.83(0.30,2.24)$ \\
\hline & & & & & 1 & $0.76(0.27,2.16)$ \\
\hline \multirow[t]{3}{*}{ BDE-99 } & No & $0.85(0.37,1.93)$ & $1 \mathrm{a}$ or $1 \mathrm{~b}$ & $0.75(0.30,1.89)$ & $\mathrm{X}$ & $0.63(0.15,2.62)$ \\
\hline & Yes & $0.52(0.16,1.77)$ & 2,3 or 4 & $0.74(0.28,1.92)$ & 0 & $0.86(0.32,2.32)$ \\
\hline & & 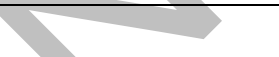 & & & 1 & $0.67(0.24,1.89)$ \\
\hline \multirow[t]{3}{*}{ BDE-100 } & $\mathrm{No}$ & $0.86(0.37,1.98)$ & $1 \mathrm{a}$ or $1 \mathrm{~b}$ & $1.14(0.45,2.89)$ & $\bar{X}$ & $0.63(0.15,2.66)$ \\
\hline & Yes & $0.98(0.30,3.23)$ & 2,3 or 4 & $0.67(0.25,1.79)$ & 0 & $1.48(0.53,4.09)$ \\
\hline & & & & & 1 & $0.62(0.22,1.79)$ \\
\hline \multirow[t]{3}{*}{ BDE-153 } & No & $0.98(0.43,2.24)$ & $1 \mathrm{a}$ or $1 \mathrm{~b}$ & $0.80(0.32,2.00)$ & $\mathrm{X}$ & $0.92(0.23,3.71)$ \\
\hline & Yes & $0.35(0.10,1.27)$ & 2,3 or 4 & $0.73(0.28,1.92)$ & 0 & $1.37(0.51,3.72)$ \\
\hline & & & & & 1 & $0.38(0.13,1.15)$ \\
\hline \multirow[t]{3}{*}{ BDE-154 } & No & $0.47(0.14,1.60)$ & $1 \mathrm{a}$ or $1 \mathrm{~b}$ & $0.84(0.33,2.11)$ & $\bar{X}$ & $1.23(0.29,5.18)$ \\
\hline & Yes & $0.93(0.41,2.14)$ & 2,3 or 4 & $0.72(0.27,1.89)$ & 0 & $1.36(0.5,3.71)$ \\
\hline & & & & & 1 & $0.34(0.11,1.04)$ \\
\hline \multirow[t]{3}{*}{ BDE-209 } & No & $2.70(1.10,6.61)^{*}$ & $1 \mathrm{a}$ or $1 \mathrm{~b}$ & $3.22(1.16,8.94)^{*}$ & $\mathrm{X}$ & $4.67(0.83,26.4)$ \\
\hline & Yes & $2.44(0.69,8.68)$ & 2,3 or 4 & $2.10(0.76,5.85)$ & 0 & $3.22(1.06,9.79)^{*}$ \\
\hline & & & & & 1 & $1.88(0.64,5.54)$ \\
\hline
\end{tabular}


537 Table 4: Adjusted odds ratios by BRAF mutation status for FR exposure above the median $538 \quad(\mathrm{n}=116)$.

\begin{tabular}{|c|c|c|c|}
\hline Mixture & Individual FR & BRAF V600E Status & OR $(95 \%$ CI $)$ \\
\hline \multirow[t]{6}{*}{ Alternate BFRs } & TBB & BRAF (+) & $0.73(0.28,1.93)$ \\
\hline & & BRAF (-) & $0.92(0.27,3.18)$ \\
\hline & & BRAF not assessed & $0.37(0.12,1.18)$ \\
\hline & TBPH & $\operatorname{BRAF}(+)$ & $1.70(0.62,4.69)$ \\
\hline & & BRAF (-) & $1.51(0.41,5.57)$ \\
\hline & & BRAF not assessed & $0.75(0.25,2.25)$ \\
\hline \multirow[t]{12}{*}{ PFRs } & ТPHP & $\operatorname{BRAF}(+)$ & $1.61(0.59,4.40)$ \\
\hline & & BRAF (-) & $5.63(1.18,26.8)^{*}$ \\
\hline & & BRAF not assessed & $1.86(0.60,5.78)$ \\
\hline & TDCIPP & $\operatorname{BRAF}(+)$ & $1.09(0.41,2.92)$ \\
\hline & & BRAF (-) & $2.01(0.55,7.41)$ \\
\hline & & BRAF not assessed & $1.93(0.63,5.86)$ \\
\hline & TCEP & $\operatorname{BRAF}(+)$ & $2.03(0.73,5.65)$ \\
\hline & & BRAF (-) & $3.76(0.89,15.9)$ \\
\hline & & BRAF not assessed & $2.35(0.76,7.31)$ \\
\hline & TCPP & $\operatorname{BRAF}(+)$ & $1.03(0.38,2.80)$ \\
\hline & & $\operatorname{BRAF}(-)$ & $1.01(0.29,3.55)$ \\
\hline & & BRAF not & $0.74(0.25,2.19)$ \\
\hline \multirow[t]{15}{*}{ Penta-BDE } & BDE-47 & $\operatorname{BRAF}(+)$ & $0.88(0.33,2.34)$ \\
\hline & & BRAF (-) & $1.16(0.33,4.00)$ \\
\hline & & BRAF not a & $0.56(0.19,1.68)$ \\
\hline & BDE-99 & $\operatorname{BRAF}(+)$ & $0.70(0.26,1.86)$ \\
\hline & & BRAF (-) & $1.75(0.49,6.25)$ \\
\hline & & BRAF not assessed & $0.46(0.15,1.40)$ \\
\hline & BD & BRAF (+) & $1.10(0.41,2.95)$ \\
\hline & & BRAF (-) & $1.75(0.49,6.29)$ \\
\hline & & BRAF not assessed & $0.43(0.14,1.33)$ \\
\hline & BDE-153 & $\operatorname{BRAF}(+)$ & $0.97(0.37,2.54)$ \\
\hline & & BRAF (-) & $1.70(0.48,6.06)$ \\
\hline & & BRAF not assessed & $0.33(0.10,1.07)$ \\
\hline & BDE-154 & $\operatorname{BRAF}(+)$ & $1.09(0.41,2.90)$ \\
\hline & & BRAF (-) & $1.25(0.36,4.42)$ \\
\hline & & BRAF not assessed & $0.40(0.13,1.24)$ \\
\hline \multirow[t]{3}{*}{ Deca-BDE } & BDE-209 & $\operatorname{BRAF}(+)$ & $1.84(0.66,5.15)$ \\
\hline & & $\operatorname{BRAF}(-)$ & $14.2(1.63,123)^{*}$ \\
\hline & & BRAF not assessed & $1.42(0.47,4.28)$ \\
\hline
\end{tabular}




\section{Figure Legend}

$541 \quad$ Figure 1:

542 Box plots of FR concentrations (ng/g dust) by case status $(n=116)$. Outliers are not shown in 543 plots. 
Figure 1: Box plots of FR concentrations (ng/g dust) by case status $(n=116)$. Outliers are not shown in plots.
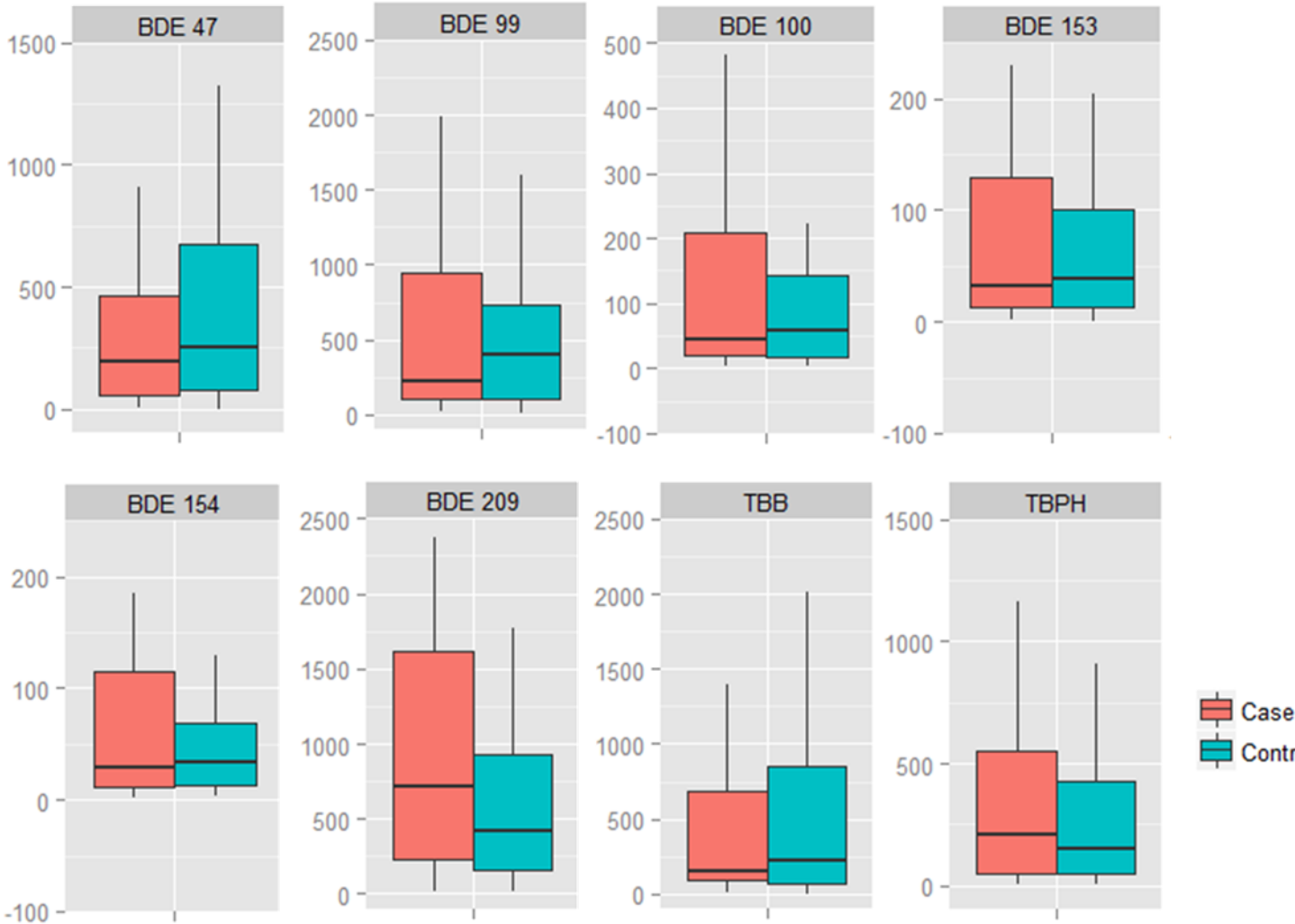

宁 Control
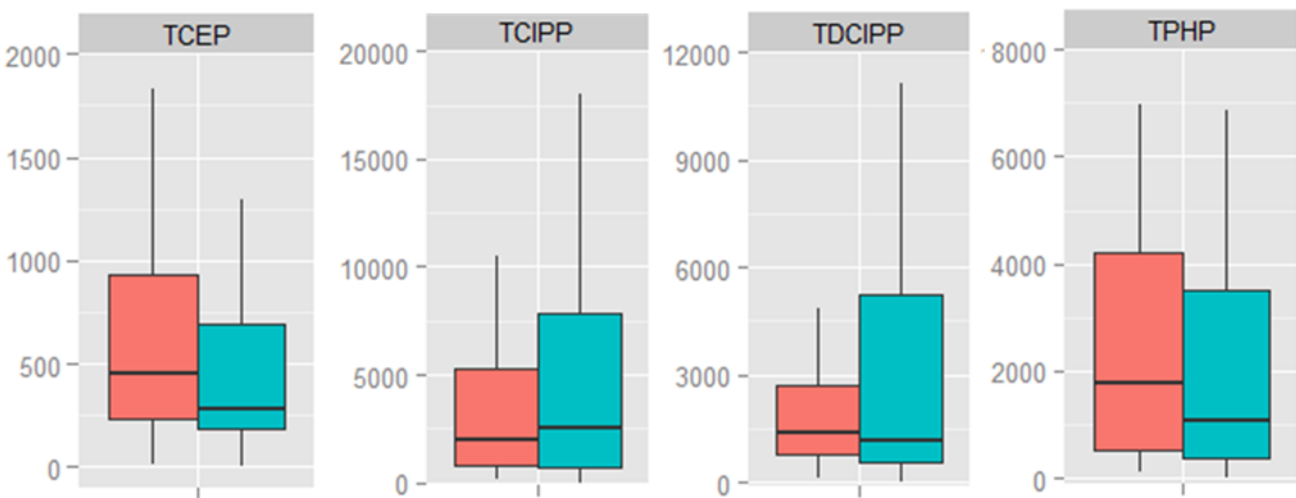


\section{Supplemental Material}

\section{EXPOSURE TO FLAME RETARDANT CHEMICALS AND THE OCCURRENCE AND SEVERITY OF PAPILLARY THYROID CANCER: A CASE-CONTROL STUDY}

Kate Hoffman, ${ }^{1}$ Amelia Lorenzo, ${ }^{1}$ Craig M. Butt, ${ }^{1}$ Stephanie C. Hammel, ${ }^{1}$ Brittany Bohinc Henderson, ${ }^{2}$ Sanziana A. Roman, ${ }^{3}$ Randall P. Scheri, ${ }^{3}$ Heather M. Stapleton, Julie Ann Sosa ${ }^{4}$

${ }^{1}$ Nicholas School of the Environment, Duke University, Durham NC 27708; ${ }^{2}$ Division of Endocrinology, Diabetes, and Metabolism, Department of Internal Medicine, Wake Forest University Baptist Medical Center and Wake Forest Comprehensive Cancer Center, Winston-Salem, NC 27157; ${ }^{3}$ School of Medicine and Department of Surgery, Duke University Medical Center, Durham, NC 27710; ${ }^{4}$ Departments of Surgery and Medicine, Duke Cancer Institute and Duke Clinical Research Institute, Duke University Medical Center, Durham, NC 27710

\section{Correspondence:}

Julie Ann Sosa

Box DUMC 2945, Durham, NC 27710

Tel +1 919-668-1767

Fax +1 919-684-6044

Email julie.sosa@duke.edu 
Supplemental Table 1: Spearman Correlations between FRS in household dust $(n=116)$

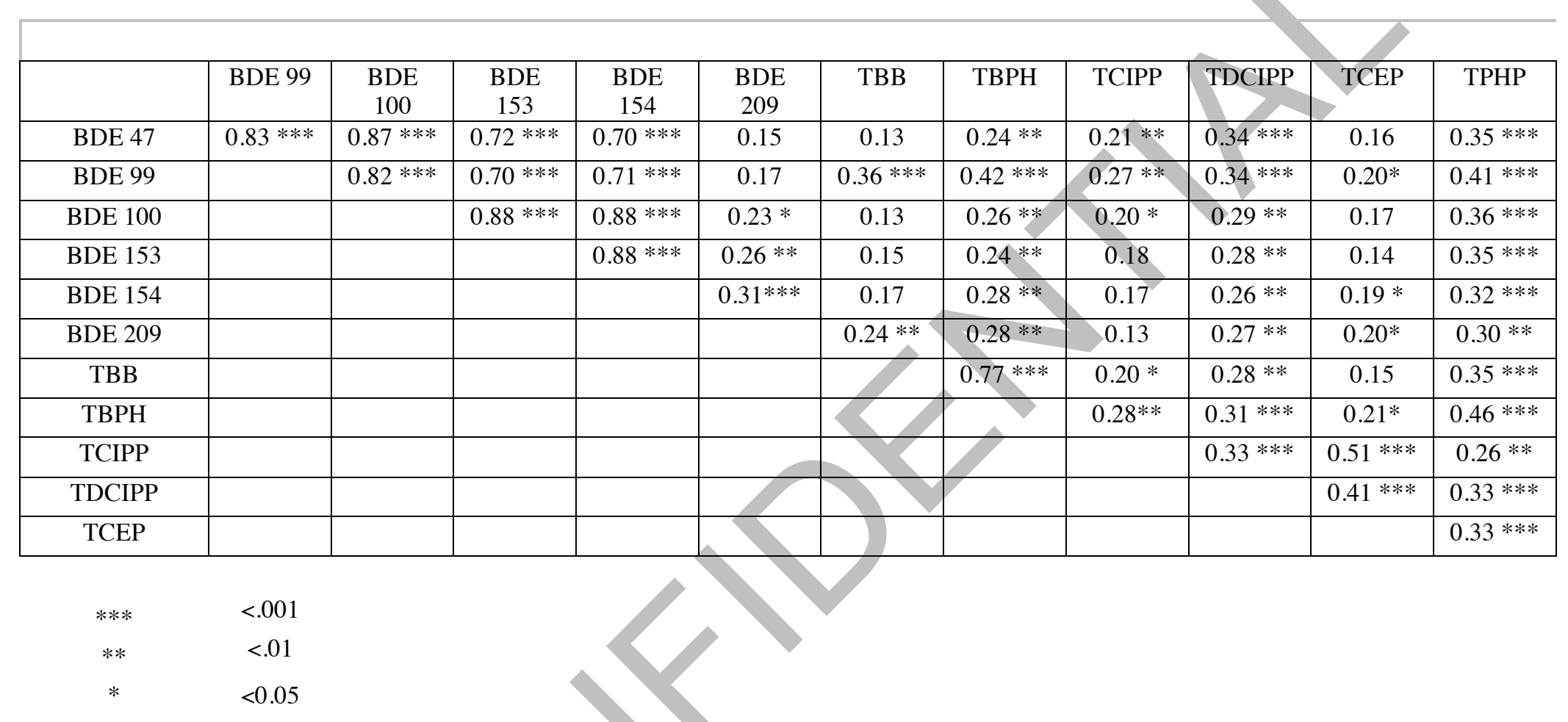




\section{Supplemental Table 2:}

Adjusted odds ratios by AJCC Stage for FR exposure above the median $(n=110)$.

\begin{tabular}{|c|c|c|}
\hline Compound & AJCC Stage & OR $(95 \% \mathrm{CI})$ \\
\hline \multirow[t]{2}{*}{ TBB } & Stage 1 & $0.78(0.33,1.85) \mathrm{p}=0.58$ \\
\hline & Stage $2-4$ & $0.38(0.11,1.29) \mathrm{p}=0.12$ \\
\hline \multirow[t]{2}{*}{ ТВРН } & Stage 1 & $1.09(0.45,2.63) \mathrm{p}=0.85$ \\
\hline & Stage $2-4$ & $1.53(0.47,4.94) \mathrm{p}=0.48$ \\
\hline \multirow[t]{2}{*}{ TDCIPP } & Stage 1 & $1.08(0.44,2.62) p=0.87$ \\
\hline & Stage 2-4 & $4.13(1.11,15.3) \mathrm{p}=0.03$ \\
\hline \multirow[t]{2}{*}{ TCEP } & Stage 1 & $2.31(0.93,5.78) \mathrm{p}=0.07$ \\
\hline & Stage $2-4$ & $3.05(0.88,10.6) \mathrm{p}=0.08$ \\
\hline \multirow[t]{2}{*}{ TCPP } & Stage 1 & $1.06(0.44,2.57) \mathrm{p}=0.90$ \\
\hline & Stage 2-4 & $0.88(0.28,2.69) \mathrm{p}=0.82$ \\
\hline \multirow[t]{2}{*}{ TPP } & & $2.02(0.82,5.01) \mathrm{p}=0.13$ \\
\hline & & $2.56(0.75,8.71) \mathrm{p}=0.13$ \\
\hline \multirow[t]{2}{*}{ BDE-47 } & & $0.83(0.35,1.98) \mathrm{p}=0.67$ \\
\hline & & $0.64(0.21,1.98) \mathrm{p}=0.44$ \\
\hline \multirow[t]{2}{*}{ BDE-99 } & Stage 1 & $0.38(0.11,1.24) \mathrm{p}=0.11$ \\
\hline & Stage 2-4 & $0.99(0.42,2.34) \mathrm{p}=0.98$ \\
\hline \multirow[t]{2}{*}{ BDE-100 } & Stage 1 & $0.96(0.40,2.31) \mathrm{p}=0.93$ \\
\hline & Stage $2-4$ & $0.82(0.27,2.52) \mathrm{p}=0.73$ \\
\hline \multirow[t]{2}{*}{ BDE-153 } & Stage 1 & $0.98(0.41,2.31) \mathrm{p}=0.95$ \\
\hline & Stage $2-4$ & $0.48(0.15,1.51) \mathrm{p}=0.21$ \\
\hline \multirow[t]{2}{*}{ BDE-154 } & Stage 1 & $1.07(0.44,2.55) \mathrm{p}=0.89$ \\
\hline & Stage $2-4$ & $0.41(0.13,1.33) \mathrm{p}=0.14$ \\
\hline \multirow[t]{2}{*}{ BDE-209 } & Stage 1 & $2.29(0.91,5.74) \mathrm{p}=0.08$ \\
\hline & Stage $2-4$ & $3.97(1.06,14.9) \mathrm{p}=0.04$ \\
\hline
\end{tabular}


Supplemental Table 3: Adjusted odds ratios for PTC and by BRAFV600E mutation status for FR exposure above the median $(n=116)$.

\begin{tabular}{lclc}
\multicolumn{2}{c}{ Serum BDE-47 } & \multicolumn{2}{c}{ Serum BDE-153 } \\
\hline Outcome & OR (95\% CI) & Outcome & OR (95\% CI) \\
\hline PTC & $0.75(0.34,1.63)$ & PTC & $0.68(0.31,1.54)$ \\
BRAF $(+)$ & $0.58(0.21,1.60)$ & BRAF $(+)$ & $0.99(0.38,2.84)$ \\
BRAF (-) & $1.06(0.28,4.04)$ & BRAF $(-)$ & $0.32(0.08,1.37)$ \\
BRAF not assessed & $0.83(0.29,2.39)$ & BRAF not assessed & $0.70(0.23,2.11)$ \\
\hline
\end{tabular}


Supplemental Figure 1: Study population consort.

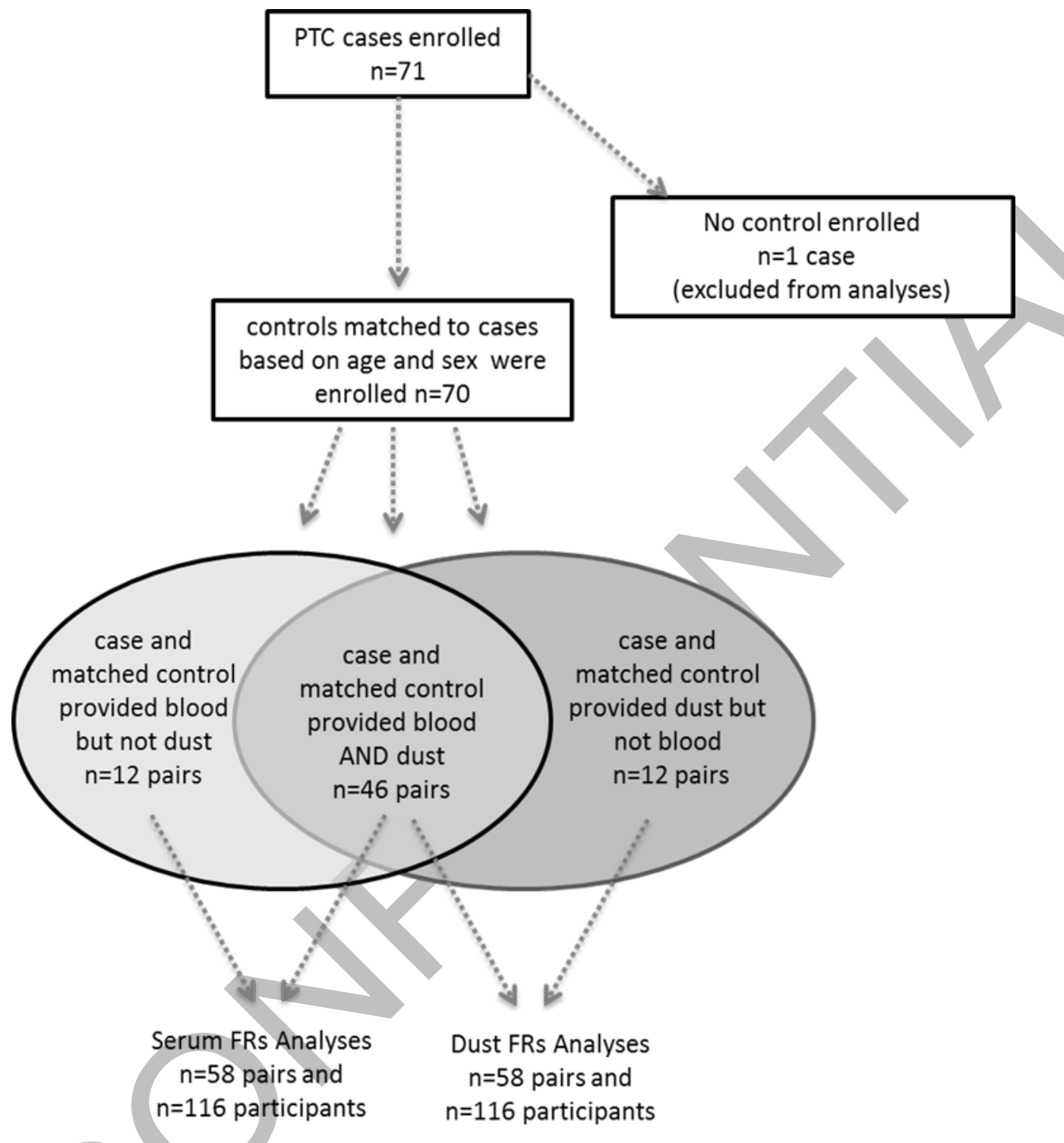

